\title{
MULTI-CRITERIONAL CHOICE OF AN ALTERNATIVE UNDER THE RULES OF FUZZY PRODUCTS WITH SOME RELIABILITY DEGREE
}

\author{
Ibrahim Abulfaz, Habibov \\ Department of Engineering Drawing ${ }^{l}$ \\ h.ibo@mail.ru \\ Oleg Dyshin \\ Scientific Research Institute Geotechnological Problems of Oil, Gas and Chemistry \\ 224 Dilara Aliyeva str., Baku, Azerbaijan, AZ 1010 \\ oleg.dyshin@mail.ru \\ Sevda Alipasha Aghammadova \\ Department of Mechanical Engineering and Materials Science ${ }^{l}$ \\ Sevda-sevda-adna@mail.ru \\ Irada Sabir Hasanzade \\ Department of General and Applied Mathematics ${ }^{l}$ \\ irada.s.hasanzade@gmail.com \\ ${ }^{1}$ Azerbaijan State Oil and Industry University \\ 20 Azadlig ave., Baku, Azerbaijan, AZ 1010
}

\begin{abstract}
One of the main problems of decision-making tasks is the need to take into account subjective expert assessments, the complete consistency of which is rare, and the choice of the best alternative. The complexity of the connections between the many-sided aspects of the decision-making situation and the lack of an accurate forecast of the consequences leads to the fact that when assessing and choosing alternatives, it is possible, and often necessary, to use and process qualitatively fuzzy estimates. In decision-making situations, when at least one of the elements (outcomes, criteria, preferences, expert opinions, etc.) is described qualitatively, indistinctly, there are problems of multi-criteria decision-making with fuzzy initial information.

Let's consider the solution to the problem of multi-criteria choice based on the rules of fuzzy conditional inference, which have the form of fuzzy statements, the conditions and conclusions of which, along with expert assessments of the criteria, are presented in the form of interval fuzzy numbers of the second type (IT2FN). The convolution of private implications in each statement is made according to Lukasiewicz's rule. To reduce the type and defuzzify the resulting IT2FN, the Karrnik-Mendel algorithm was used to construct the minimum and maximum centroids of nested fuzzy sets of the first type, which give an estimate of the utility interval for each alternative. To refine the obtained utility estimates, under conditions of incomplete definiteness of statements, using the generalized Bayesian inference mechanism, adjusted estimates of the utility intervals of alternatives are constructed. By comparing these intervals, a larger interval is determined and the corresponding alternative is taken as a solution to the problem under consideration.

The application of the proposed approach to solving the problem of multicriteria selection of the most corroded section of a gas pipeline with ambiguous expert opinions is shown. To date, specific practical and theoretical results have been obtained for decision-making problems with fuzzy initial information.
\end{abstract}

Keywords: subjective expert assessment, multi-criteria choice of an alternative, Bayesian inference mechanism, expert opinion.

DOI: $10.21303 / 2461-4262.2021 .001708$

\section{Introduction}

The solution of problems of multicriteria choice in conditions of uncertainty of the correspondence of alternatives to the requirements of criteria can be made, in particular, using the rules of fuzzy conditional inference, in the form of the so-called fuzzy production rules (FPR) [1-3].

In this case, the basis of the decision support system is the knowledge base formed by a set of rules of the type «if <condition>, then <conclusion>». In this case, the conditional part and the conclusion are fuzzy statements that are formalized by fuzzy sets, while the construction of the FPR is carried out in an expert way. 
In the general case, the search for the best solution from the set of feasible ones consists in processing a certain set of FPRs describing the situation. Following [4, 5], let's assume that $U$ is the set of alternatives $u_{j}, j=1, \ldots, N ; X$ is the set of criteria $X_{\ell}, \ell=1, \ldots, q$ and $E$ is the set of rules (statements) $d_{k}, k=1, \ldots, p$. Moreover, it is assumed that $X_{\ell}$ is a linguistic variable with terms $X_{t \ell, \ell}$, $t_{\ell}=1, \ldots, v_{\ell}$ on the base set $U_{\ell} \subseteq U$ and, in addition, the criterion $X_{\ell}$ corresponds to the fuzzy set:

$$
X_{\ell}=x_{1 \ell} / u_{1}+x_{2 \ell} / u_{2}+\ldots+x_{N \ell} / u_{N}
$$

where the numbers $x_{j \ell} \in[0,1], j=1, \ldots, N$ characterize the degree of satisfaction of the criterion $X_{\ell}$ of the alternative $u_{j}$ and are determined by an expert.

In general, the statement $d_{k c}$ has the form $d_{k}$ : «if $X_{1}=A_{1 k}$ and $X_{2}=A_{2}$ and $X_{q}=A_{q k}$, then $Y=B_{k}$ », and the $\ell$-th part of the condition $X_{\ell k}=A_{\ell k}$ can consist of $m_{\ell k}$ subconditions $X_{\ell k}=A_{\ell k, 1}$ or $A_{\ell k, 2}$ or... $A_{\ell k, m \ell k}$. Then, writing $d_{k}$ in the form $d_{k}$ : «X=A $A_{k}$, then $Y=B_{k} »$, and denoting the degree of truth of the subconditions with the $A_{\ell k, m \ell k}\left(m_{\ell k}=1, \ldots, m_{\ell k}^{0}\right)$ by $\mu_{\ell k, m}$, let's obtain:

$$
\mu_{A_{k}}(v)=\min _{\ell}\left(\max _{m \ell k} \mu_{\ell k, m \ell k}\left(u_{\ell}\right)\right), v \in V=U_{1} \times U_{2} \times \ldots U_{q} .
$$

Here $X=X_{1} \times X_{2} \times \ldots \times X_{q}\left(\times\right.$ is the sign of the Cartesian product), $A_{\ell k, m \ell k} \in T_{\ell}\left(T_{\ell}\right.$ is the set of terms of the linguistic variable $\left.(L V) X_{\ell} ; T_{\ell}=\left(X_{\ell 1}, X_{\ell 2}, \ldots, X_{\ell v}, \ldots, X_{\ell v \ell}\right)\right) ; A_{\ell k}=A_{\ell k, 1} \cup A_{\ell k, 2} \cup \ldots \cup A_{\ell k, m \ell k}$ is the union of some union $m_{\ell k} \in v_{\ell}$ of terms from $T_{\ell}$.

$Y$ is a linguistic variable characterizing the idea of the decision maker (DM) about the satisfactory (acceptability) of a set of several criteria with the corresponding values and having the terms $Y_{t Y}, t_{Y}=1, \ldots, v_{Y} ; B_{k}$ is one of the terms.

Assuming that $X_{\ell \ell}, X_{t \ell \ell}\left(t_{\ell}=1, \ldots, v_{\ell}, \ell=1, \ldots, q\right)$ and $Y_{t_{y}}\left(t_{Y}=1, \ldots, v_{Y}\right)$ are fuzzy sets of the first type (T1FS), the multicriteria alternative choice method was developed in $[4,5]$. The numerical implementation of this method is shown in [3] using the example of the problem of selecting candidates for a vacant position at the faculty of the institute.

In multi-criteria tasks related to technical, environmental, medical and other types of risks, the main goal is to identify various emergencies. To solve this problem, systematic monitoring surveys of the state of controlled objects are carried out, as a result of which some rules and patterns of abnormal behavior leading to a particular emergency situation are developed. These rules are formulated in the form of statements, the conditions of which include a set of criteria values (symptom complex), and in the conclusions, the degree of confidence in the diagnosed situation. On the basis of the obtained knowledge base, the rules of fuzzy products with linguistic input and output variables are constructed. Further, using the fuzzy inference algorithm (FID), the degree of confidence in the diagnosis is calculated as a numerical coefficient as a result of defuzzification of the corresponding fuzzy inference. This approach with the use of the fuzzy inference algorithm [6] in [7] solved one of the main tasks to ensure the reliability of the gas pipeline operation. The numerical procedure for implementing the algorithm, detailed in [7], can serve as the basis for the mathematical support of the diagnostic decision support system using fuzzy logic.

A generalization of the method $[4,5]$ to the case of specifying the initial information in the form of interval fuzzy sets of the second type (IT2FN) was obtained in $[8,9]$ with a numerical implementation.

It should be noted that the general decision support system based on the interval fuzzy sets of the second type (IT2FS) introduced by Zadeh [10] using the fuzzy inference algorithm and containing inputs, $m$ outputs and $N$ rules of fuzzy productions is considered in [11-16]. The lower and upper utility estimates $y_{j}$ and $\bar{y}_{j}$ obtained in these works for the $j$-th output linguistic variable $(L V)$, only allow to judge the degree of acceptability of the entire system. At the same time, the acceptability of each constituent element of the system remains uncertain, since the problem of choosing the most acceptable alternative (system element) has not been solved.

The most general approach to the problem of multicriteria choice, using the technique of ordering solutions by similarity to the ideal solution, was proposed in [17-19]. This approach uses fuzzy networks and aggregation of production rules and is applied, in particular, in the case of 
specifying the initial information in the form of IT2FN, but requires much more computational operations compared to the method described in $[4,5]$.

Both in [3-5, 8, 9] and in [11-19], the problem of multicriteria choice of an alternative was actually solved provided that all statements were fully confirmed. In this paper, we have developed a method for solving the problem of multicriteria choice when specifying the initial information in the form of IT2FN and interval estimates of the factors of certainty of statements. In this case, the estimates of the utility intervals of the alternatives obtained as a result of the application of the Karnik-Mendel algorithm [20] are corrected using the generalized interval Bayesian inference mechanism [21, 22]. By comparing the intervals obtained by the method [23], the largest interval is found.

\section{Methods of research}

2. 1. Multi-criteria choice of an alternative based on reliable rules of fuzzy productions

Let's consider the method of multicriteria choice of alternatives described in $[4,5]$ based on the compositional rule of aggregation of expert assessments of the degrees of satisfaction of criteria by alternatives with reliable information about the preferences of the decision maker (DM), given in the form of fuzzy judgments.

Let the set of solutions be characterized by a set of criteria $X_{1}, X_{2}, \ldots, X_{q}$, which are linguistic variables on the base sets $U_{1}, U_{2}, \ldots, U_{q}$, where $U_{\ell} \subseteq U=\left(u_{1}, \ldots, u_{N}\right) \ell=1, \ldots q ; U_{j}, j=1, \ldots, N$ - alternatives. Let $T_{\ell}$ denote the set of terms $X_{\ell v \ell}\left(v=1, \ldots, v_{\ell}\right.$ of the linguistic variable $X_{\ell}$, i. e. $X_{\ell v \ell}=x_{1, \ell v \ell} / u_{1}+x_{2, \ell v \ell} / u_{2}+\ldots+x_{N, \ell v \ell} / u_{N}$. The derivation of each statement $d_{k}(k=1, \ldots, p)$ with the output linguistic variable $Y$, defined by the set $T_{0}$ of terms $Y_{v}\left(v=1, \ldots, v_{0}\right)$ characterizes the decision maker's idea of the satisfaction (acceptability) of the set of several criteria included in the statement condition with some fixed values of their terms corresponding to this statement.

In the general case, the statement (fuzzy production rule (FPR)) $d_{k}$ has the form:

$$
d_{k} \text { : if } X_{1}=A_{k, 1} \text { and } X_{2}=A_{k, 2} \text { and } \ldots \text { and } X_{q}=A_{k, q} \text {, then } Y=B_{k} \text {, }
$$

where $A_{k \ell}$ is one of the terms $X_{\ell v}, \ell=1, \ldots, q$.

Let's denote the intersection $X_{1}=A_{k, 1} \cap X_{2}=A_{k, 2} \cap \ldots \cap X_{q}=A_{k, q}$ by $X=A_{k}$, then rule (1) can be written as a fuzzy implication:

$$
d_{k} \text { if } X=A_{k} \text {, then } Y=B_{k} \text {. }
$$

In this case $A_{k}=A_{k, 1} \cap A_{k, 2} \cap \ldots \cap A_{k, q}, B_{k} \in[0,1]$ :

$$
\underline{\mu}_{A_{k}}(v)=\min \left(\underline{\mu}_{A_{k}, 1}\left(u_{1}\right), \underline{\mu}_{A_{k}, 2}\left(u_{2}\right), \underline{\mu}_{A_{k}, q}\left(u_{q}\right)\right), v=V
$$

where $V=U_{1} \times U_{2} \times \ldots \times U_{q} ; v=\left(u_{1}, u_{2}, \ldots, u_{q}\right)$.

Let's denote the base set $U$ or $V$ by $W$. Then $A_{k}$ is a fuzzy subset in $W$, and $B_{k}$ is a fuzzy subset in $[0,1]$. In the calculations, the set $[0,1]$ is discretized into the set $I=(0,0.1,0.2, \ldots, 1)$.

The implication of fuzzy sets (2) in the sense of Lukasiewicz is expressed as follows:

$$
\mu_{H_{k}}(w, i)=\min \left(1,\left(1-\mu_{A_{K}}(w)+\mu_{B_{k}}(i)\right)\right),
$$

where $H_{k}$ is a fuzzy subset on $W \times I, w \in W, i \in I$.

The intersection of the sets $H_{k}$ gives a functional solution $D=H_{1} \cap H_{2} \cap \ldots \cap H_{p}$, so for each $(w, i) \in W \times I$ :

$$
\mu_{D}(w, i)=\min _{k=1, \ldots, p}\left(\mu_{H_{k}}(w, i)\right)
$$

where $p$ is the total number of statements and each row in matrix $D$ is T1FS in $W \times I$. 
Let alternative $j$ be described by a fuzzy subset $U_{j}$ in $W$. Satisfaction of the alternative $u_{j}$ is based on the compositional rule of fuzzy inference:

$$
E_{j}=U_{j} \circ D
$$

where $E_{j}$ is a fuzzy subset defined on $I$. Then:

$$
\mu_{E_{j}}(i)=\max _{w \in W}\left(\min \left(\mu_{U_{j}}(w), \mu_{D}(w, i)\right) .\right.
$$

If each $U_{j}$ consists of only one element, i. e. $U_{j}=\left\{u_{j}\right\}$, then $U_{j}$ is a singleton, and (7) takes the form:

$$
\mu_{E_{j}}=\mu_{D}\left(U_{j}, i\right)
$$

In other words, $E_{j}$ is the $j$-th row in the $N \times 11$ matrix $D$, since the discrete set $I=(0,0.1,0.2, \ldots, 1)$ consists of 11 elements.

Obviously, for interval estimates $\left(a_{\ell}^{(j)}, b_{\ell}^{(j)}\right)$ degrees of satisfaction of criteria $X_{\ell}$ by alternatives $u_{j}(j=1, \ldots, N)$, each of $X_{\ell}$ is a linguistic variable with terms $X_{\ell v \ell}$, represented by IT2FN. This means that each term $X_{\ell v \ell}$ corresponds to two fuzzy sets of type 1 (T1FS):

$$
\begin{aligned}
& \underline{X}_{\ell v_{\ell}}=\underline{x}_{1, \ell v_{\ell}} / u_{1}+\underline{x}_{2, \ell v_{\ell}} / u_{2}+\ldots+\underline{x}_{N, \ell v_{\ell}} / u_{N}, \\
& \bar{X}_{\ell v_{\ell}}=\bar{x}_{1, \ell v_{\ell}} / u_{1}+\bar{x}_{2, \ell v_{\ell}} / u_{2}+\ldots+\bar{x}_{N, \ell v_{\ell}} / u_{N},
\end{aligned}
$$

moreover, $x_{j, \ell v \ell} \leq \bar{x}_{j, \ell v \ell}(j=1, \ldots, N)$. As a result, let's obtain a representation of $A_{k}$ in the form of IT2FN with a lower membership function (MF):

$$
\underline{\mu}_{A_{k}}(v)=\min \left(\underline{\mu}_{A_{k}, 1}\left(u_{1}\right), \underline{\mu}_{A_{k}, 2}\left(u_{2}\right), \underline{\mu}_{A_{k}, q}\left(u_{q}\right)\right), \quad v=V .
$$

And upper MF:

$$
\bar{\mu}_{A_{k}}(v)=\min \left(\bar{\mu}_{A_{k}, 1}\left(u_{1}\right), \bar{\mu}_{A_{k}, 2}\left(u_{2}\right), \bar{\mu}_{A_{k}, q}\left(u_{q}\right)\right), \quad v=V
$$

and if:

$$
A_{k, \ell}=X_{\ell, v_{k \ell}}, \quad v_{k \ell} \in\left(1, \ldots, v_{\ell}^{0}\right)
$$

then $\underline{\mu}_{A_{k}, \ell}\left(u_{j}\right)=\underline{x}_{j, \ell v_{k \ell}}$ and $\bar{\mu}_{A_{k}, \ell}\left(u_{j}\right)=\bar{x}_{j, \ell v_{k \ell}}$.

In interval estimates of the degrees of satisfaction of the criteria $X_{\ell}$ alternatives $u_{j}$ $(j=1, \ldots, N)$, the $L V$ terms $X_{\ell}$ and, therefore, the sets $A_{k}$ in the FPR $d_{k}(k=1, \ldots, p)$ become IT2FN. In this case, in formulas (4), (5) T1FS $A_{k}$ and $B_{k}$ are replaced by IT2FN $A_{k}$ and $B_{k}$, and the functional solution $D$ for each fixed $u \in U$ is IT2FN, which composition with the singleton $U_{j}=\left\{u_{j}\right\}$ is IT2FN, which characterizes satisfactory alternative $j$. This set corresponds to the maximum $\left(\bar{E}_{j}\right)$ and minimum $\left(E_{j}\right)$ fuzzy sets of the first type obtained by the composition $U_{j}$ with the fuzzy sets of the first type $\bar{D}$ and $D$, respectively:

$$
\bar{D}=\bar{H}_{1} \cap \bar{H}_{2} \cap \ldots \cap \bar{H}_{p} \text { and } \underline{D}=\underline{H}_{1} \cap \underline{H}_{2} \cap \ldots \cap \underline{H}_{p}
$$

where $H_{k}$ and $\bar{H}_{k}$ are obtained by implication (4) with $A_{k}, B_{k}$ replaced by $A_{k}, B_{k}$ and $\overline{A_{k}}, \bar{B}_{k}$, respectively. T1FS $E_{j}$ and $D$ are replaced by $\tilde{E}_{j}$ and $\tilde{D}$, respectively. Moreover, $\tilde{E}_{j}$ is the $j$-th row in the $(N \times 11)$ matrix $\tilde{D}$.

Thus, we obtain a generalization of the method $[4,5]$ for solving the multicriteria problem of choosing alternatives to the case of specifying the input variables $X_{\ell}(\ell=1, \ldots, q)$ and the output variable $Y$ by interval fuzzy numbers of the second type. 
Defuzzification of each IT2FN $\tilde{E}_{j}$ is done using a centroid. In this case, the boundaries of the interval $\left[y_{\ell}, y_{r}\right]$ are calculated using the iterative Karrnik-Mendel algorithm or using the approximation formula for the center $\varepsilon$ and the length J corresponding to IT2FS [11]. As a result of this [20], let's find, $y_{\ell}=\varepsilon-J, y_{r}=\varepsilon+J$, where:

$$
\begin{gathered}
\varepsilon=\sum_{i=1}^{N} h_{i} x_{i} / \sum_{i=1}^{N} h_{i}, \quad J=\sum_{i=1}^{N}\left|x_{i}-\varepsilon\right| \cdot \Delta_{i}, x_{i}=0.1 \cdot i, i=0,1, \ldots, N, N=10 . \\
J_{x_{i}}=\left[L_{i}, R_{i}\right], \quad h_{i}=\left(L_{i}+R_{i}\right) / 2, \quad \Delta_{i}=\left(R_{i}-L_{i}\right) / 2 .
\end{gathered}
$$

Comparison of intervals $\left(y_{\ell}^{(j)}, y_{r}^{(j)}\right)(j=1, \ldots, N)$ is performed by one of the well-known methods for comparing clear intervals. As proved in [23], the largest of the intervals in the set of intervals $\left(y_{\ell}^{(j)}, y_{r}^{(j)}\right)(j=1, \ldots, N)$ is the interval with the largest center $\bar{y}^{(j)}=\left(y_{\ell}^{(j)}, y_{r}^{(j)}\right) / 2$. Let this be the interval corresponding to the number $j=j^{*}$. Then the alternative $u_{j}$ is a solution to the problem of multicriteria choice of an alternative formed at the beginning of Section 2 with the most acceptable estimate of the best acceptability interval $\left(y_{\ell}^{\left(j^{*}\right)}, y_{r}^{\left(j^{*}\right)}\right)$.

\section{2. Application of generalized interval Bayesian inference mechanisms to the prob- lem of multicriteria choice of an alternative}

In the previous section, the problem of multicriteria choice of an alternative was considered in the case of specifying the initial information both by fuzzy sets of the first type (T1FS) and by interval fuzzy numbers of the second type (IT2FN). In both cases, the rules for fuzzy productions were assumed to be reliable. However, expert opinions, on the basis of which these rules are developed, are somehow extrapolated and must take into account the possible impact of changes in the external environment and market characteristics. Therefore, usually in expert judgments $d_{k}$ there is a certain factor of certainty of statements (evidence) $v\left(d_{k}\right), 0<v\left(d_{k}\right) \leq 1$, which requires correcting the degrees of reliability of the hypotheses $u=u_{j}$ obtained earlier under the assumption that $v\left(d_{k}\right)=1$, i. e., under the condition that the statements $d_{k}$ are categorical.

For this purpose, the work proposes to use interval Bayesian inference mechanisms [21, 22].

The problem of multicriteria choice of an alternative based on fuzzy logical inference with given factors of certainty of statements $0<v\left(d_{k}\right) \leq 1$ is solved in 2 stages. At the first stage, assuming $v\left(d_{k}\right)=1$ for all $k=1, \ldots, p$, the problem of multicriteria choice of an alternative is solved by the above method. At the second stage, the obtained interval estimates $\left(y_{\ell}^{\left(j^{*}\right)}, y_{r}^{\left(j^{*}\right)}\right)$ of the adequacy (acceptability) of the hypothesis $u_{j}$ are corrected using interval Bayesian inference mechanisms.

The main idea of the Bayesian approach is the sequential use of Bayes' theorem to calculate a posteriori estimates of the probabilities of hypotheses, taking into account a certain set of associated evidence (judgments). In this case, the a posteriori estimates of the probabilities obtained at the previous step act as a priori at the next one.

Let the connection between hypotheses $H=\left\{h_{j}\right\}_{j=1}^{N}$ and evidence $E=\left\{e_{k}\right\}_{k=1}^{M}$ be expressed using production rules of the form «if $e$, then $h »$. Each of them is associated with the value of the function $\delta: V \rightarrow R^{2}$ (the strength of the rule) defined on the set $V \subseteq E \times H$ of all pairs of interrelated evidence and hypotheses as follows: $\delta(e, h)=(\hat{P}(e / h), \hat{P}(e / \bar{h}))$. Here $\bar{h}$ is the negation of the hypothesis $h, \hat{P}(e / h)$ and $\hat{P}(e / \bar{h})$ are the standard notation of conditional probabilities accepted in probability theory. For expert estimates of the corresponding values (in the case of $h=u, e=d$ ), the conditional probabilities $\hat{P}(e / h)$ and $\hat{P}(e / \bar{h})$ can be obtained from the relations:

$$
\begin{aligned}
& \widehat{P}(e / h) \cdot \widehat{P}(h)=\widehat{P}(h / e) \cdot \widehat{P}(e), \\
& \widehat{P}(e / \bar{h}) \cdot \hat{P}(\bar{h})=\hat{P}(\bar{h} / e) \cdot \widehat{P}(e) .
\end{aligned}
$$

When specifying the point estimates $B(h), v(e)(0 \leq v(e) \leq 1)$ and conditional probabilities $\hat{P}(e / h)$ and $\hat{P}(e / \bar{h})$, recalculating the reliability estimate $B(h)$ of hypothesis $\mathrm{h}$ taking into account the influence of the certificate $\mathrm{e}$ is produced according to the formula: 
where

$$
B(h):=Q(\delta(e, h), B(h), v(e))
$$

$$
\begin{gathered}
Q(\delta(e, h), B(h), u(e))=B(h)+B(h)(1-B(h))(\widehat{P}(e / h)-\widehat{P}(e / \bar{h}) v(e)) K(\delta(e, h), B(h)), \\
K(\delta(e, h), B(h))=\frac{1}{B(h) \hat{P}(e / h)+(1-B(h)) \hat{P}(e / \bar{h})}
\end{gathered}
$$

he estimates $\hat{P}(e / h)$ and $\hat{P}(e / \bar{h})$ are calculated using the Bayes formula:

$$
\widehat{P}(e / h)=\frac{\widehat{P}(h / e) \cdot \widehat{P}(e)}{B(h)},
$$

where

$$
B(\bar{h} / e)=1-B(h), \hat{P}(e / \bar{h})=1-\widehat{P}(h / e) .
$$

In the case of interval specified estimates:

$$
B^{L}(h) \leq B(h) \leq B^{R}(h), \hat{P}^{L}(h / e) \leq \hat{P}(h / e) \leq \hat{P}^{R}(h / e), \quad 0 \leq v^{L}(e) \leq v(e) \leq v^{R}(e) \leq 1,
$$

let's define the lower and upper bounds for the conditional probabilities $P(e / h), P(e / \bar{h})$, and $P(\bar{h} / e)$ as follows:

$$
\begin{gathered}
\hat{P}^{L}(e / h)=\frac{\hat{P}^{L}(h / e) \cdot \hat{P}(e)}{\widehat{B}^{R}(h)}, \hat{P}^{R}(e / h)=\frac{\widehat{P}^{R}(h / e) \cdot \hat{P}(e)}{\hat{B}^{L}(h)}, \\
\hat{P}^{L}(e / \bar{h})=\frac{\hat{P}^{L}(\bar{h} / e) \cdot \hat{P}(e)}{\hat{B}^{R}(\bar{h})}, \hat{P}^{R}(e / \bar{h})=\frac{\hat{P}^{R}(\bar{h} / e) \cdot \widehat{P}(e)}{\hat{B}^{L}(\bar{h})}, \\
\hat{P}^{L}(\bar{h} / e)=1-\hat{P}^{R}(h / e), \hat{P}^{R}(\bar{h} / e)=1-\hat{P}^{L}(h / e) .
\end{gathered}
$$

The iterative procedure for recalculating the validity of each hypothesis $h_{j}$ will be performed sequentially. Let's start with the evidence $e=e_{1}$ and $B_{0}^{L}\left(h_{j}\right)=y_{\ell}^{(j)}$ and $B_{0}^{R}\left(h_{j}\right)=y_{r}^{(j)}$, where $y_{\ell}^{(j)}$ and $y_{r}^{(j)}$, estimating the satisfactory interval of the alternative $u_{j}$. The process ends with a testimony $e=e_{M}$ ( $M$ is the total number of testimonials-statements). As estimates of the conditional probabilities $P^{L}\left(h_{j} / e_{k}\right)$ and $P^{R}\left(h_{j} / e_{k}\right)$ for $h_{j}=u_{j}, e_{k}=d_{k}$, the values:

$$
\begin{aligned}
& P^{L}\left(u_{j} / d_{k}\right)=\min \left(\underline{\mu}_{X_{1}, v_{k 1}}\left(u_{j}\right), \underline{\mu}_{X_{2}, v_{k 2}}\left(u_{j}\right), \ldots, \underline{\mu}_{X_{q}, v_{k q}}\left(u_{j}\right)\right), \\
& P^{R}\left(u_{j} / d_{k}\right)=\min \left(\bar{\mu}_{X_{1}, v_{k 1}}\left(u_{j}\right), \bar{\mu}_{X_{2}, v_{k 2}}\left(u_{j}\right), \ldots, \bar{\mu}_{X_{q}, v_{k q}}\left(u_{j}\right)\right) .
\end{aligned}
$$

The results of the last ( $M$-th) step $B_{M}^{L}\left(h_{j}\right)$ and $B_{M}^{R}\left(h_{j}\right)$ are taken as the left and right boundaries of the satisfactory interval for the hypothesis $h_{j}$. The procedure is repeated for the remaining $h_{j}$ from the set $H=\left\{h_{j}\right\}_{j=1}^{N}$.

In the case of interval estimates using an iterative procedure, three cases are distinguished [22]:

1) $\hat{P}^{R}\left(e_{k} / h_{j}\right) \leq \hat{P}^{L}\left(e_{k} / \bar{h}_{j}\right)$;

2) $P^{L}\left(e_{k} / h_{j}\right) \geq P^{R}\left(e_{k} / \bar{h}_{j}\right)$;

3) $\widehat{P}^{R}\left(e_{k} / h_{j}\right)>\widehat{P}^{L}\left(e_{k} / \bar{h}_{j}\right)$ and $\hat{P}^{R}\left(e_{k} / \bar{h}_{j}\right)>\widehat{P}^{L}\left(e_{k} / h_{j}\right)$. 
Introducing the notation:

$$
\begin{aligned}
& a_{1}=\widehat{P}^{R}\left(h_{i} / e_{k}\right) / B_{k-1}^{L}\left(h_{j}\right), \quad b_{1}=\left(1-\widehat{P}^{R}\left(h_{j} / e_{k}\right)\right) /\left(1-B_{k-1}^{L}\left(h_{j}\right)\right), \\
& a_{2}=\hat{P}^{L}\left(h_{j} / e_{k}\right) / B_{k-1}^{R}\left(h_{j}\right), \quad b_{2}=\left(1-\widehat{P}^{L}\left(h_{j} / e_{k}\right)\right) /\left(1-B_{k-1}^{R}\left(h_{j}\right)\right)
\end{aligned}
$$

and determining at each iteration step $k$ the lower and upper bounds for the conditional probabilities $P\left(e_{k} / h_{j}\right)$ and $P\left(e_{k} / \bar{h}\right)$ as follows:

$$
\begin{gathered}
\hat{P}^{L}\left(e_{k} / h_{j}\right)=\frac{\hat{P}^{L}\left(h_{j} / e_{k}\right) \cdot \hat{P}\left(e_{k}\right)}{\hat{B}_{k-1}^{R}\left(h_{j}\right)}, \hat{P}^{R}\left(e_{k} / h_{j}\right)=\frac{\hat{P}^{R}\left(h_{j} / e_{k}\right) \cdot \hat{P}\left(e_{k}\right)}{\hat{B}_{k-1}^{L}\left(h_{j}\right)}, \\
\hat{P}^{L}\left(e_{k} / \bar{h}_{j}\right)=\frac{\hat{P}^{L}\left(\bar{h}_{j} / e_{k}\right) \cdot \widehat{P}\left(e_{k}\right)}{\hat{B}_{k-1}^{R}\left(\bar{h}_{j}\right)}, \hat{P}^{R}\left(e_{k} / \bar{h}_{j}\right)=\frac{\widehat{P}^{R}\left(\bar{h}_{j} / e_{k}\right) \cdot \widehat{P}\left(e_{k}\right)}{\hat{B}_{k-1}^{L}\left(\bar{h}_{j}\right)},
\end{gathered}
$$

where

$$
\begin{gathered}
\hat{P}^{L}\left(\bar{h}_{j} / e_{k}\right)=1-\hat{P}^{R}\left(h_{j} / e_{k}\right), \hat{P}^{R}\left(\bar{h}_{j} / e_{k}\right)=1-\hat{P}^{L}\left(h_{j} / e_{k}\right), \\
\hat{B}_{k}^{L}\left(\bar{h}_{j}\right)=1-\widehat{B}^{R}\left(h_{j}\right), \hat{B}_{k}^{R}\left(\bar{h}_{j}\right)=1-\widehat{B}^{L}\left(h_{j}\right),
\end{gathered}
$$

recurrent formulas for calculating $B_{k}^{L}\left(h_{j}\right)$ and $B_{k}^{R}\left(h_{j}\right)$ in cases 1), 2), and 3) are written, respectively, as:

$$
\begin{aligned}
& \left.B_{k}^{L}\left(h_{j}\right)=B_{k-1}^{L}\left(h_{j}\right)\left[1+\frac{\left(a_{2}-b_{2}\right)\left(1-B_{k-1}^{L}\left(h_{j}\right)\right)}{a_{2} B_{k-1}^{L}\left(h_{j}\right)+b_{2}\left(1-B_{k-1}^{L}\left(h_{j}\right)\right)} \cdot v^{R}\left(e_{k}\right)\right]\right] \\
& \left.B_{k}^{R}\left(h_{j}\right)=B_{k-1}^{R}\left(h_{j}\right)\left[1+\frac{\left(a_{1}-b_{1}\right)\left(1-B_{k-1}^{R}\left(h_{j}\right)\right)}{a_{1} B_{k-1}^{R}\left(h_{j}\right)+b_{1}\left(1-B_{k-1}^{R}\left(h_{j}\right)\right)} \cdot v^{L}\left(e_{k}\right)\right]\right\} \text {, } \\
& B_{k}^{L}\left(h_{j}\right)=B_{k-1}^{L}\left(h_{j}\right)\left[1+\frac{\left(a_{2}-b_{2}\right)\left(1-B_{k-1}^{L}\left(h_{j}\right)\right)}{a_{2} B_{k-1}^{L}\left(h_{j}\right)+b_{2}\left(1-B_{k-1}^{L}\left(h_{j}\right)\right)} \cdot v^{L}\left(e_{k}\right)\right] \\
& \left.B_{k}^{R}\left(h_{j}\right)=B_{k-1}^{R}\left(h_{j}\right)\left[1+\frac{\left(a_{1}-b_{1}\right)\left(1-B_{k-1}^{R}\left(h_{j}\right)\right)}{a_{1} B_{k-1}^{R}\left(h_{j}\right)+b_{1}\left(1-B_{k-1}^{R}\left(h_{j}\right)\right)} \cdot v^{R}\left(e_{k}\right)\right]\right\} \text {, } \\
& B_{k}^{L}\left(h_{j}\right)=B_{k-1}^{L}\left(h_{j}\right)\left[1+\frac{\left(a_{2}-b_{2}\right)\left(1-B_{k-1}^{L}\left(h_{j}\right)\right)}{a_{2} B_{k-1}^{L}\left(h_{j}\right)+b_{2}\left(1-B_{k-1}^{L}\left(h_{j}\right)\right)} \cdot v^{R}\left(e_{k}\right)\right] \\
& \left.B_{k}^{R}\left(h_{j}\right)=B_{k-1}^{R}\left(h_{j}\right)\left[1+\frac{\left(a_{1}-b_{1}\right)\left(1-B_{k-1}^{R}\left(h_{j}\right)\right)}{a_{1} B_{k-1}^{R}\left(h_{j}\right)+b_{1}\left(1-B_{k-1}^{R}\left(h_{j}\right)\right)} \cdot v^{R}\left(e_{k}\right)\right]\right\} \text {. }
\end{aligned}
$$

As can be seen, all formulas in (25)-(27) do not depend on $\hat{P}\left(e_{k}\right)$, therefore, the inequalities and describing the above three cases, for $\hat{P}\left(e_{k}\right) \neq 0$, will, according to (16)-(18), be equivalent to the following inequalities:

$$
\frac{\hat{P}^{L}\left(h_{j} / e_{k}\right)}{B_{k-1}^{L}\left(h_{j}\right)} \leq \frac{1-\hat{P}^{R}\left(h_{j} / e_{k}\right)}{1-B_{k-1}^{L}\left(h_{j}\right)} ;
$$




$$
\begin{aligned}
& \frac{\hat{P}^{L}\left(h_{j} / e_{k}\right)}{B_{k-1}^{R}\left(h_{j}\right)} \geq \frac{1-\hat{P}^{L}\left(h_{j} / e_{k}\right)}{1-B_{k-1}^{R}\left(h_{j}\right)} ; \\
& \frac{\hat{P}^{R}\left(h_{j} / e_{k}\right)}{B_{k-1}^{L}\left(h_{j}\right)}>\frac{1-\hat{P}^{L}\left(h_{j} / e_{k}\right)}{1-B_{k-1}^{L}\left(h_{j}\right)}
\end{aligned}
$$

and

$$
\frac{1-\hat{P}^{L}\left(h_{j} / e_{k}\right)}{1-B_{k-1}^{R}\left(h_{j}\right)}>\frac{\hat{P}^{L}\left(h_{j} / e_{k}\right)}{B_{k-1}^{R}\left(h_{j}\right)} .
$$

For the initial approximation of the lower $B_{0}^{L}\left(h_{j}\right)$ and upper $B_{0}^{R}\left(h_{j}\right)$ estimates of the degree of satisfaction of the hypothesis $h_{j}$, it is possible to take, respectively, the numbers $y_{\ell}^{(j)}$ and $y_{r}^{(j)}$ obtained in Section 2.1 as a result of solving the multicriteria choice problem for categorical statements.

For a fixed $j$, calculations by formulas (25), (26), and (27) should be carried out only for those evidence $e_{k}$ for which the condition is satisfied, in this case:

$$
B_{k}^{L}\left(h_{j}\right)=B_{k-1}^{L}\left(h_{j}\right) \text { and } B_{k}^{R}\left(h_{j}\right)=B_{k-1}^{R}\left(h_{j}\right) .
$$

It follows from (16), (17) that the lower and upper bounds for the conditional probabilities $P(e / h)$ and $P(e / \bar{h})$ are expressed at each $k$-th step up to the factor $\hat{P}\left(e_{k}\right)$ in terms of known quantities. Therefore, they are easily compared without the availability of information about the estimates of $\hat{P}\left(e_{k}\right)$. In addition, the recurrent calculation formulas (25), (26), and (27) are also independent of the estimate $P\left(e_{k}\right)$.

The result of this iterative procedure is the intervals $\left(\hat{y}_{\ell}^{(j)}, \hat{y}_{r}^{(j)}\right)$ of the refined estimates of the satisfiability of the hypotheses $u_{j}(j=1, \ldots, N)$. By comparing in the general case of overlapping intervals, let's find the largest interval $\left(\hat{y}_{\ell}^{\left(j^{*}\right)}, \hat{y}_{r}^{\left(j^{*}\right)}\right)$ among all the intervals in the population $\left(\hat{y}_{\ell}^{(j)}, \hat{y}_{r}^{(j)}\right)(j=1, \ldots, N)$. Therefore, the alternative $u=u_{j^{*}}$ is the best. As proved in [15], the largest among a given set of intervals is the interval with the largest center $\bar{y}^{(j)}=\left(\hat{y}_{\ell}^{(j)}, \hat{y}_{r}^{(j)}\right) / 2$.

\section{Research results}

The effectiveness of the proposed method for solving the problem of multicriteria choice of an alternative, given the factors of certainty of statements, is illustrated by the example of choosing the most corroded section of a gas pipeline.

Let's consider 4 sections $\left(j_{0}=4\right)$ of the Kazy-Magomed-Kazakh gas pipeline: $62-70 \mathrm{~km}(j=1)$; $134-135 \mathrm{~km}(j=2) ; 139-140.5 \mathrm{~km}(j=3)$ and $154-155.5 \mathrm{~km}(j=4)$, for which expert lower $a_{\ell}^{(j)}$ and upper $b_{\ell}^{(j)}$ estimates of the change in feature $\ell$ for object $j$ are given (Table 1), on the basis of which, by the membership functions of the terms $X_{\ell, v \ell}$ of the features $\ell(\ell=1,2, \ldots, 5), v_{\ell}=1, \ldots, v_{\ell}^{0}$, it is easy to calculate taking into account the weight coefficients $\omega_{\ell}$, the «lower» $\alpha_{\ell, \nu \ell}^{(j)}$ and «upper» $\beta_{\ell, \nu \ell}^{(j)}$ estimates of the membership function of the term $X_{\ell, v \ell}$ for object $j$ :

$$
\alpha_{\ell, v_{\ell}}^{(j)}=\omega_{\ell} \cdot \mu_{\ell, v_{\ell}}\left(a_{\ell}^{(j)}\right), \beta_{\ell, v_{\ell}}^{(j)}=\omega_{\ell} \cdot \mu_{\ell, v_{\ell}}\left(b_{\ell}^{(j)}\right),
$$

where $\mu_{\ell, v e}(x)$ is the membership function of the term $X_{\ell, v \ell}$, while $\mu_{\ell, v \ell}(x)=0$ if $x \notin \operatorname{supp} X_{\ell, v \ell}$ $\left(\operatorname{supp} X_{\ell, v \ell}\right.$ is the support of the term $X_{\ell, v \ell}$, that is, the set of points $x$ for which $\left.\mu_{\ell, v \ell}(x) \neq 0\right)$.

Based on (31), each term $X_{\ell, v \ell}$ of the variable $X_{\ell}$ is IT2FN $X_{\ell, v \ell}$, to which there correspond two T1FS defined on the discrete base set $U=\left(u_{1}, u_{2}, u_{3}, u_{4}\right)$ and presented, respectively, in the form:

$$
\begin{aligned}
\overline{\tilde{X}}_{\ell, v} & =\left\{\beta_{\ell, v}^{(1)} / u_{1}, \beta_{\ell, v}^{(2)} / u_{2}, \beta_{\ell, v}^{(3)} / u_{3}, \beta_{\ell, v}^{(4)} / u_{4}\right\}, \\
\underline{X}_{\ell, v} & =\left\{\alpha_{\ell, v}^{(1)} / u_{1}, \alpha_{\ell, v}^{(2)} / u_{2}, \alpha_{\ell, v}^{(3)} / u_{3}, \alpha_{\ell, v}^{(4)} / u_{4}\right\} .
\end{aligned}
$$


Table 1

Expert assessments of changes in a characteristic

\begin{tabular}{|c|c|c|c|c|c|c|}
\hline Site location & $\begin{array}{c}\text { Severity } \\
\text { of anomalies }\end{array}$ & & $\begin{array}{c}\text { Residual pipe wall } \\
\text { thickness from } \\
\text { design, in \% }\end{array}$ & $\begin{array}{l}\text { Specific electrical } \\
\text { resistance of soil, } \\
\text { Ohm·m }\left(X_{1}\right)\end{array}$ & $\begin{array}{c}\text { Potential } \\
\text { «pipe-ground», } \\
\text { in volts, }\left(X_{2}\right)\end{array}$ & $\begin{array}{c}\text { Stress level in the } \\
\text { walls of the gas } \\
\text { pipeline } \mathrm{MPa}\left(X_{3}\right)\end{array}$ \\
\hline \multirow[t]{3}{*}{$62-70 \mathrm{~km}$} & $0.814-0.845$ & I & $16.5 \pm 0.5$ & $0.63-1.56$ & $(-0.617 \ldots-0.538)$ & $1.71-1.95$ \\
\hline & $0.527-0.715$ & II & $19.2 \pm 0.5$ & & & \\
\hline & $0.114-0.279$ & III & $46.6 \pm 0.5$ & & & \\
\hline \multirow[t]{3}{*}{$134-135 \mathrm{~km}$} & $0.819-0.833$ & I & $14.8 \pm 0.5$ & $2.76-3.28$ & $(-0.525 \ldots-0.6050)$ & $1.65-1.88$ \\
\hline & $0.511-0.674$ & II & $16.7 \pm 0.5$ & & & \\
\hline & $0.09-0.195$ & III & $42.2 \pm 0.5$ & & & \\
\hline \multirow[t]{3}{*}{$139-140.5 \mathrm{~km}$} & $0.815-0.824$ & I & $14.2 \pm 0.5$ & $8.6-11.3$ & $(-0.493 \ldots-0.511)$ & $1.75-1.98$ \\
\hline & $0.582-0.688$ & II & $15.3 \pm 0.5$ & & & \\
\hline & $0.154-0.224$ & III & $40.6 \pm 0.5$ & & & \\
\hline \multirow[t]{3}{*}{$154-155.5 \mathrm{~km}$} & $0.856-0.874$ & I & $13.2 \pm 0.5$ & $12.7-16.4$ & $(-0.351 \ldots-0.533)$ & $1.6-1.95$ \\
\hline & $0.635-0.789$ & II & $14.0 \pm 0.5$ & & & \\
\hline & $0.215-0.374$ & III & $38.4 \pm 0.5$ & & & \\
\hline
\end{tabular}

Let's take the following as input variables affecting the growth rate of corrosion defects:

- $X_{1}$ is specific electrical resistance of the soil (Ohm·m);

$-X_{2}$ is redox potential of the soil - redox potential $(\mathrm{V})$;

$-X_{3}$ is the stress level in the walls of the gas pipeline (MPa);

- $X_{4}$ is the state of the metal, determined by the residual (from the design) pipe wall thickness, $\%$;

- $X_{5}$ is the degree of danger of anomalies $(1-F)$.

In accordance with the degree of influence of the variables $X_{1}, X_{2}, X_{3}, X_{4}, X_{5}$ on the occurrence and development of corrosion, let's take into account their weight coefficients in the calculations:

$$
\omega_{1}=0.12 ; \quad \omega_{2}=0.08 ; \quad \omega_{3}=0.25 ; \quad \omega_{4}=0.35 ; \quad \omega_{5}=0.20, \quad \sum_{i=1}^{5} \omega_{i}=1 .
$$

To determine the operability of areas with defects, it is necessary to control the gas pipeline throughout. This also evaluates the limiting state due to perforating corrosion or cracking. In addition, it will be possible to propose plans for the alternate repair of sections of the main gas pipeline (MGP). Therefore, as an output variable, as in [7], let's choose «Potentially predicted corrosion rate (PPCR)», taking into account the monotonic increase of this parameter from the variable «Potentially predicted corrosion rate (PPCR)».

For the input variables $X_{1}, \ldots, X_{5}$, let's use the following sets of terms, respectively: $T_{1}=$ (low, medium, high); $T_{2}=$ (very low, low, medium, high); $T_{3}=\left(\right.$ low, medium, high, high); $T_{4}=(\mathrm{bad}$, average, good); $T_{5}=($ low, medium, high). A graphical representation of the terms of input variables is given in [7]. For the output variable $Y$, the following terms are used: $T_{0}=$ (low, moderate, medium, elevated, high), which are replaced, respectively, by terms: unsatisfactory (US), almost satisfactory (AS), satisfactory (S), more than satisfactory (MS), very satisfactory (VS). Each of the terms of $L V Y$ is defined in the form of INMT2, which have a lower and upper membership function at $x=I=\{0 ; 0.1 ; 0.2 ; \ldots ; 1\}$. Wherein:

$$
\begin{gathered}
\underline{\mu}_{S}(x)=x \sqrt{x}, \bar{\mu}_{S}(x)=x ; \underline{\mu}_{U S}(x)=1-x ; \bar{\mu}_{U S}(x)=1-x \sqrt{x} ; \\
\underline{\mu}_{M S}(x)=x^{2} ; \bar{\mu}_{M S}(x)=x \sqrt{x} ; \underline{\mu}_{A S}(x)=1-x \sqrt{x} ; \bar{\mu}_{A S}(x)=1-x^{2} ; \\
\underline{\mu}_{V S}(x)=x^{3} ; \bar{\mu}_{V S}(x)=x^{2} .
\end{gathered}
$$


To assess the corrosion rate, let's use the following fuzzy production rules (FPR):

$$
\begin{gathered}
d_{1}: \text { if } X=X_{3,1} \text {, then } Y=U S ; d_{2}: \text { if } X=X_{3,2}, \text { then } Y=A S ; \\
d_{3}: \text { if } X=X_{3,3} \text {, then } Y=S ; d_{4}: \text { if } X=X_{3,4} \text {, then } Y=M S ; \\
d_{5}: \text { if } X=X_{1,1} \text {, then } Y=S ; d_{6}: \text { if } X=X_{1,2}, \text { then } Y=A S ; \\
d_{7}: \text { if } X=X_{1,3} \text {, then } Y=U S ; d_{8}: \text { if } X=X_{2,4} \text {, then } Y=U S ; \\
d_{9}: \text { if } X=X_{2,3}, \text { then } Y=S ; d_{10}: \text { if } X=X_{2,2} \text {, then } Y=M S ; \\
d_{11}: \text { if } X=X_{2,1}, \text { then } Y=V S ; d_{12}: \text { if } X=X_{5,3} \text {, then } Y=V S ; \\
d_{13}: \text { if } X=X_{5,2}, \text { then } Y=S ; d_{14}: \text { if } X=X_{5,1}, \text { then } Y=U S ; \\
d_{15}: \text { if } X=X_{4,3} \text { and } X_{5,1}, \text { then } Y=U S ; d_{16}: \text { if } X=X_{4,2}, \text { and } X_{5,2} \text { then } Y=S ; \\
\quad d_{17}: \text { if } X=X_{4,1} \text { and } X_{5,3} \text {, and } Y=V S .
\end{gathered}
$$

At the first stage of solving the multicriteria problem of choosing an alternative, after performing calculations in accordance with the method described in Section 2. 1, let's obtain:

$$
\begin{aligned}
& \begin{array}{c||ccccccccccc||}
\overline{\tilde{D}}=u_{1} & 0 & 0.1 & 0.2 & 0.3 & 0.4 & 0.5 & 0.6 & 0.7 & 0.8 & 0.9 & 1 \\
u_{2} & 0.8 & 0.81 & 0.97 & 1 & 1 & 1 & 1 & 1 & 1 & 0.9 & 0.75 \\
u_{3} & 0.8 & 0.81 & 1 & 1 & 1 & 1 & 1 & 1 & 1 & 0.9 & 0.75 \\
u_{4} & 0.8 & 0.81 & 1 & 1 & 1 & 1 & 1 & 1 & 1 & 0.9 & 0.75 \\
0.8 & 0.81 & 1 & 1 & 1 & 1 & 1 & 1 & 1 & 0.9 & 0.75
\end{array} \\
& \underline{\tilde{\tilde{D}}=} \begin{array}{c||ccccccccccc||}
u_{1} & 0 & 0.1 & 0.2 & 0.3 & 0.4 & 0.5 & 0.6 & 0.7 & 0.8 & 0.9 & 1 \\
u_{2} & 0.65 & 0.68 & 0.74 & 0.81 & 0.9 & 1 & 1 & 1 & 0.975 & 0.875 & 0.725 \\
u_{3} & 0.65 & 0.68 & 0.74 & 0.81 & 0.9 & 1 & 1 & 1 & 0.950 & 0.850 & 0.750 \\
u_{4} & 0.68 & 0.74 & 0.81 & 0.9 & 1 & 1 & 1 & 0.975 & 0.875 & 0.750 \\
0.65 & 0.68 & 0.74 & 0.81 & 0.9 & 1 & 1 & 1 & 0.950 & 0.850 & 0.750 \| .
\end{array}
\end{aligned}
$$

Let $\bar{d}_{j, i}$ and и $d_{j, i}(i=0,1, \ldots, 10)$ denote the $j$-th row in the $(4 \times 11)$ matrices $\overline{\bar{D}}$ and $\tilde{\tilde{D}}$, respectively. Then for each $j(j=1, \ldots, 4)$ let's obtain an interval fuzzy set of the second type (INMT2), for which the $j$-row in the matrix $\overline{\tilde{D}}$ and $\tilde{\tilde{D}}$ is, respectively, the $\underset{\tilde{\tilde{E}}}{\operatorname{maximum}}$ and minimum fuzzy sets of the first type embedded in the INMT2. Let's denote them by $\overline{\tilde{E}}_{j}$ and $\tilde{\tilde{E}}_{j}$, respectively.

Using the estimates of the parameters $\varepsilon$ and $J$ for IT1FS approximating IT2FS [20] to reduce the type and defuzzification of the INMT2 $\tilde{E}_{j}(j=1, \ldots, 4)$, let's find the following estimates for the intervals of satisfactory hypotheses $u_{j}$ :

$$
\begin{gathered}
{\left[y_{\ell}^{(1)}, y_{r}^{(1)}\right]=[0.41,0.71], \quad\left[y_{\ell}^{(2)}, y_{r}^{(2)}\right]=[0.47,0.61],} \\
{\left[y_{\ell}^{(3)}, y_{r}^{(3)}\right]=[0.497,0.523], \quad\left[y_{\ell}^{(4)}, y_{r}^{(4)}\right]=[0.496,0.524],}
\end{gathered}
$$

having, respectively, centers $\bar{y}^{(1)}=0.56, \bar{y}^{(2)}=0.54, \bar{y}^{(3)}=0.51, \bar{y}^{(4)}=0.1$.

Comparing the intervals $\left[y_{\ell}^{(j)}, y_{r}^{(j)}\right](j=1, \ldots, 4)$ according to the method [23], let's come to the conclusion that the alternative $u=u_{1}$. is the best. This conclusion is obtained provided that all statements are categorical. 
At the second stage, the multicriteria problem of choosing an alternative is solved for non-categorical statements with given certainty $0 \leq v^{L}\left(d_{k}\right) \leq v\left(d_{k}\right) \leq v^{R}\left(d_{k}\right) \leq 1,(k=1, \ldots, p)$, where $v^{L}\left(d_{k}\right)=0.8$ and $v^{R}\left(d_{k}\right)=0.9$. By applying an iterative Bayesian inference procedure, let's get the adjusted estimates.

$$
\begin{aligned}
& {\left[y_{\ell}^{(1)}, y_{r}^{(1)}\right]=[0.470,0.610], \quad\left[y_{\ell}^{(2)}, y_{r}^{(2)}\right]=[0.497,0.523],} \\
& {\left[y_{\ell}^{(3)}, y_{r}^{(3)}\right]=[0.496,0.524], \quad\left[y_{\ell}^{(4)}, y_{r}^{(4)}\right]=[0.183,0.369],}
\end{aligned}
$$

having centers respectively:

$$
\stackrel{-(1)}{y}=0.54, \quad \stackrel{-(2)}{y}=0.51, \quad \stackrel{-(3)}{y}=0.508, \quad \stackrel{-(4)}{y}=0.194 .
$$

Thus, the use of the Bayesian inference mechanism makes it possible to obtain an unambiguous ranking of alternatives according to their satisfaction.

\section{Discussion of the research results on the multicriteria choice of an alternative in case of ambiguous expert opinions}

The initial data for solving the problem with statements $d[k]$, given uncategorically with factors of certainty $0<\gamma[k]<1$, were given in the following Table 2.

\begin{tabular}{|c|c|c|c|c|c|c|c|c|}
\hline \multirow{2}{*}{$\bar{D}$} & \multicolumn{4}{|c|}{$P^{L}[j, k]$} & \multicolumn{4}{|c|}{$P^{R}[j, k]$} \\
\hline & $j=1$ & $j=2$ & $j=3$ & $j=4$ & $j=1$ & $j=2$ & $j=3$ & $j=4$ \\
\hline 1 & 0.225 & 0.25 & 0.225 & 0.25 & 0.25 & 0.25 & 0.25 & 0.25 \\
\hline 2 & 0.225 & 0.05 & 0.25 & 0.025 & 0.237 & 0.212 & 0.25 & 0.27 \\
\hline 3 & 0 & 0 & 0 & 0 & 0 & 0 & 0 & 0 \\
\hline 4 & 0 & 0 & 0 & 0 & 0 & 0.12 & 0 & 0 \\
\hline 5 & 0.35 & 0.35 & 0.35 & 0.35 & 0.12 & 0 & 0 & 0 \\
\hline 6 & 0 & 0 & 0.12 & 0 & 0 & 0.12 & 0.12 & 0 \\
\hline 7 & 0 & 0 & 0 & 0.12 & 0 & 0 & 0 & 0 \\
\hline 8 & 0.08 & 0 & 0 & 0.12 & 0.08 & 0.08 & 0 & 0 \\
\hline 9 & 0.008 & 0.08 & 0.08 & 0.08 & 0.01 & 0.08 & 0.08 & 0.08 \\
\hline 10 & 0 & 0 & 0 & 0 & 0 & 0 & 0 & 0 \\
\hline 11 & 0 & 0 & 0 & 0 & 0 & 0 & 0 & 0 \\
\hline 12 & 0 & 0 & 0 & 0 & 0.2 & 0.2 & 0.2 & 0.2 \\
\hline 13 & 0.2 & 0.2 & 0.2 & 0.2 & 0.2 & 0.2 & 0.2 & 0.2 \\
\hline 14 & 0 & 0 & 0 & 0 & 0 & 0 & 0 & 0 \\
\hline 15 & 0 & 0 & 0 & 0 & 0 & 0 & 0 & 0 \\
\hline 16 & 0 & 0 & 0 & 0 & 0 & 0 & 0 & 0 \\
\hline 17 & 0 & 0 & 0 & 0 & 0.2 & 0.2 & 0.2 & 0.2 \\
\hline
\end{tabular}

Table 2

Initial data for solving the problem

Further, it was supposed:

$$
\begin{gathered}
v^{L}[k]=0.8, v^{R}[k]=0.9, k=1, \ldots, 27 ; B^{L}[1,0]=0.41 ; B^{R}[1,0]=0.71 ; B^{L}[2,0]=0.47 ; \\
B^{R}[2,0]=0.61 ; B^{L}[3,0]=0.497 ; B^{R}[3,0]=0.523 ; B^{L}[4,0]=0.496 ; B^{R}[4,0]=0.524 .
\end{gathered}
$$

Evidence with numbers $k=5,7,8$, having implausible data for some $j=j_{0}$, were excluded from the calculation for $j=j_{0}$. 
Comparison of the calculation results under the conditions of categorical and non-categorical statements showed:

- in the first case, alternatives 1, 2, 3, 4 are ranked in the order 1 (I), 2 (II), 3 (III-IV), 4 (III-IV) (the ranks are indicated in brackets, a lower rank corresponds to a higher utility), in the second case - 1 (I), 2 (II), 3 (III), 4 (IV).

Thus, in the second case, the ranks are determined quite uniquely. The results obtained are explained by the fact that, provided that the statements are categorical, the calculation is made according to the boundary (left and right) values of the features from the specified intervals of their values. In this case, all values within these intervals are taken with a confidence level equal to 1 (in accordance with the definition of the values of the secondary membership function of interval type-2 fuzzy numbers). Under the conditions of non-categorical statements, this drawback is eliminated by introducing certainty factors $v[k], 0 \leq v^{L}[k] \leq v[k] \leq v^{R}[k] \leq 1$ for each statement $d[k]$. For simplicity, the calculations assume $v^{L}[k]=0.8$ and $v^{R}[k]=0.9$.

In the proposed method, due to the introduction of certainty factors $v[k]$ and the use of the Bayesian inference mechanism, the calculation results can also take into account the degree of competence of the experts giving the conclusions of the statements. To do this, it is enough to reflect in the estimates $v^{L}[k]$ and $v^{R}[k]$, respectively, the lower and upper estimates of the competence of experts who take part both in assessing the values of features (criteria) and in making conclusions in statements.

Some experts who assess the values of the attributes of alternatives (in our case, these are gas pipeline sections) do not take into account the attribute membership functions developed by specialists. Therefore, their estimates may not belong to the carrier of the membership function (i. e., the points at which it takes nonzero values), which leads to estimates of the conditional probabilities $D^{R}[j, k]=0$.

In this case, the statement $d_{k}$ is inactive and should be excluded from the recurrent formulas for calculating $B_{k}^{L}\left(h_{j}\right)$ and $B_{k}^{R}\left(h_{j}\right)$, which is a certain limitation of the method.

This drawback can be eliminated by using such interval estimates of experts for features that are included in the carrier of the feature membership function.

Further generalization of Bayesian mechanisms can be obtained by applying fuzzy inference. The prospects for using such methods are described in [21, 22].

\section{Conclusions}

The proposed method of multicriteria choice of an alternative is applicable in the general case of statements with given lower and upper estimates of the certainty factor.

As a result of recalculating the estimates of the usefulness of alternatives, calculated under the condition of the categoricity of each statement, using generalized interval Bayesian inference mechanisms, an unambiguous ranking of the usefulness of alternatives is obtained, which allows choosing the best one.

Due to the application of the iterative procedure for calculating the conditional probabilities of confirming the alternatives, in each individual statement of the experts, adjusted estimates of the satisfactoryness of hypotheses are obtained. When implementing this procedure, only active statements are taken into account, for which expert assessments of the features included in them belong to the corresponding membership functions, which somewhat limits the application of the method.

The multi-criteria choice of an alternative with reliable (categorical) rules of fuzzy products is based on the established (conservative) judgments of experts, formed as a result of long-term observations from the prehistory of the process. New trends in the development of the process under study lead to the need to calculate the estimates of the usefulness of the considered alternatives, taking into account the risk and uncertainty associated with the introduction of new technologies and possible changes in the estimates of utility and preferences.

Bayesian inference mechanisms are one of the main methods of adaptive selection of the best alternative when drawing up a business plan for commercial activities in any area of production and services. In this case, each alternative is compared with quantitative estimates of material and financial costs and the corresponding economic effect is calculated. 


\section{References}

[1] Pospelov, D. A. (Ed.) (1986). Nechetkie mnozhestva v modelyah upravleniya i iskusstvennogo intellekta. Moscow: Nauka, 312.

[2] Borisov, A. N., Alekseev, A. V., Merkur'eva, G. V. (1989). Obrabotka nechetkoy informatsii v sistemah prinyatiya resheniy. Moscow: Radio i svyaz', 304.

[3] Borisov, A. I., Krumberg, O. A., Fedorov, I. P. (1990). Prinyatie resheniy na osnove nechetkih modeley. Primery ispol'zovaniya. Riga: Zinatne, 184.

[4] Yager, R. R. (1977). Multiple objective decision-making using fuzzy sets. International Journal of Man-Machine Studies, 9 (4), 375-382. doi: https://doi.org/10.1016/s0020-7373(77)80008-4

[5] Yager, R. R. (1984). General multiple-objective decision functions and linguistically quantified statements. International Journal of Man-Machine Studies, 21 (5), 389-400. doi: https://doi.org/10.1016/s0020-7373(84)80066-8

[6] Loktyuhin, V. N., Mal'chenko, S. I., Cherepnin, A. A. (2009). Osnovy matematicheskogo obespecheniya podderzhki diagnosticheskih resheniy v biotekhnicheskih sistemah s ispol'zovaniem nechetkoy logiki. Ryazan': RGRTU, 64.

[7] Gabibov, I. A., Dyshin, O. A., Agammedova, S. A. (2015). Kompleksnaya otsenka skorosti korrozionnyh protsessov v gazoprovode na osnove tekhnologii nechetkoy logiki. Gazovaya promyshlennost', 724, 99-104.

[8] Dyshin, O. A., Yahyaeva, A. N. (2016). Selection based best alternative fuzzy inference interval of fuzzy sets of the second type. Herald of the Azerbaijan Engineering Academy, 8 (2), 87-95.

[9] Veliyev, R. G., Dyshin, O. A. (2016). Linguistic model of decision making process for education system based on interval fuzzy sets II. Herald of the Azerbaijan Engineering Academy, 8 (4), 68-82.

[10] Zadeh, L. A. (1975). The concept of a linguistic variable and its application to approximate reasoning-III. Information Sciences, 9 (1), 43-80. doi: https://doi.org/10.1016/0020-0255(75)90017-1

[11] Liang, Q., Mendel, J. M. (2000). Interval type-2 fuzzy logic systems: theory and design. IEEE Transactions on Fuzzy Systems, 8 (5), 535-550. doi: https://doi.org/10.1109/91.873577

[12] Castillo, O., Melin, P. (2007). 12 Design of Fuzzy Inference Systems with the Interval Type-2 Fuzzy Logic Toolbox. Studies in Fuzziness and Soft Computing, 145-154. doi: https://doi.org/10.1007/978-3-540-76284-3_12

[13] Castro, J. R., Castillo, O., Martinez, L. G. (2007). Interval Type-2 Fuzzy Logic Toolbox. Engineering Letters, 15 (1). Available at: http://www.engineeringletters.com/issues_v15/issue_1/EL_15_1_14.pdf

[14] Liu, Z.-Q., Liu, Y.-K. (2010). Type-2 fuzzy variables and their arithmetic. Soft Computing, 14 (7), 729-747. doi: https:// doi.org/10.1007/s00500-009-0461-x

[15] Kahraman, C., Öztayşi, B., Uçal Sarı, İ., Turanoğlu, E. (2014). Fuzzy analytic hierarchy process with interval type-2 fuzzy sets. Knowledge-Based Systems, 59, 48-57. doi: https://doi.org/10.1016/j.knosys.2014.02.001

[16] Mendel, J. M. (2017). Uncertain Rule-Based Fuzzy Systems. Introduction and New Directions. Springer. doi: https://doi.org/ 10.1007/978-3-319-51370-6

[17] Chen, T.-Y., Tsao, C.-Y. (2008). The interval-valued fuzzy TOPSIS method and experimental analysis. Fuzzy Sets and Systems, 159 (11), 1410-1428. doi: https://doi.org/10.1016/j.fss.2007.11.004

[18] Zhang, X., Xu, Z. (2014). Extension of TOPSIS to Multiple Criteria Decision Making with Pythagorean Fuzzy Sets. International Journal of Intelligent Systems, 29 (12), 1061-1078. doi: https://doi.org/10.1002/int.21676

[19] Yaakob, A. M., Gegov, A., Abdul Rahman, S. F. (2018). Selection of alternatives using fuzzy networks with rule base aggregation. Fuzzy Sets and Systems, 341, 123-144. doi: https://doi.org/10.1016/j.fss.2017.05.027

[20] Karnik, N. N., Mendel, J. M. (2001). Centroid of a type-2 fuzzy set. Information Sciences, 132 (1-4), 195-220. doi: https:// doi.org/10.1016/s0020-0255(01)00069-x

[21] Verevka, O. V., Zalozhenkova, I. A., Parasyuk, I. N. (2000). Interval'nye bayesovskie mekhanizmy vyvoda i ih prilozheniya. Problemy programmirovaniya, 1-2, 467-470.

[22] Verevka, O. V., Zalozhenkova, I. A., Parasyuk, I. N. (1998). Obobshchenie interval'nyh bayesovskih mekhanizmov vyvoda i perspektivy ih ispol'zovaniya. Kibernetika i sistemniy analiz, 6, 3-13.

[23] Levin, V. I. (2016). Analysis of Interval Functions by Methods of Interval Differential Calculus. Systems of Control, Communication and Security, 1, 335-350.

Received date 09.12.2020

(C) The Author(s) 2021

Accepted date 10.03.2021

Published date 31.03.2021

This is an open access article under the CC BY license (http://creativecommons.org/licenses/by/4.0).

How to cite: Habibov, I. A., Dyshin, O., Aghammadova, S. A., Hasanzade, I. S. (2021). Multi-criterional choice of an alternative under the rules of fuzzy products with some reliability degree. EUREKA: Physics and Engineering, 2, 124-136. doi: https://doi.org/ 10.21303/2461-4262.2021.001708 